\title{
High temperature resins based on allylamine/bismaleimides
}

\author{
King-Fu Lin*, Jin-Sing Lin and Chen-Hwa Cheng \\ Institute of Materials Science and Engineering, National Taiwan University, Taipei, Taiwan \\ 10617, Republic of China \\ (Received 8 January 1996; revised 14 January 1996)
}

\begin{abstract}
Synthesis, curing, and physical/mechanical properties of $4,4^{\prime}$-bismaleimidodiphenylmethane (BDM), $4,4^{\prime}$ bismaleimidodiphenylether (BDE), 3,3'-bismaleimidodiphenylsulfone (3-BDS), and 4,4' -bismaleimidodiphenylsulfone (4-BDS) adducted with various amount of allylamines were investigated and compared with each other. BDM was reacted with allylamines exclusively through the Michael addition reaction, whereas 3-BDS and 4-BDS were reacted with allylamines by amidation along with the cleavage of an imide-ring $\mathrm{C}-\mathrm{N}$ bond. Only BDE underwent both reactions to yield BDE/allylamine adducts. Three types of curing reactions might occur depending on the amount of adducted allylamines: (1) thermal homopolymerization through the maleimide double bonds; (2) accelerated homopolymerization by allylamines; and (3) polymerization of the cleaved allylamines by themselves or with the maleimido groups. Of all the BMIs/ allylamines adducts under study, the cured $\mathrm{BDM} / 50 \mathrm{~mol} \%$ allylamine and $\mathrm{BDE} / 50 \mathrm{~mol} \%$ allylamine adducts have superior mechanical properties. In addition, the former resin has a glass transition temperature $\left(T_{\mathrm{g}}\right)$ of $335^{\circ} \mathrm{C}$ and a degradation temperature $\left(T_{\mathrm{d}}\right)$ of $471^{\circ} \mathrm{C}$, whereas the latter has a $T_{\mathrm{g}}$ of $349^{\circ} \mathrm{C}$ and a $T_{\mathrm{d}}$ of $436^{\circ} \mathrm{C}$. Copyright (C) 1996 Elsevier Science Ltd.
\end{abstract}

(Keywords: bismaleimides; allylamines; high temperature resins)

\section{INTRODUCTION}

Bismaleimide (BMI) resins have been developed most rapidly among the polyimides during the past two decades. The main aim of the development was to offer high temperature performance, high toughness, low cost, and maintain the epoxy-like processing. According to the recent review paper by Pater ${ }^{1}$, BMIs have been modified by five different methods: (1) synthesis of BMIs with flexible or long segments in the backbone $e^{2,3} ;$ (2) use of aromatic diamine as a chain extender to react with BMIs through the Michael addition reaction ${ }^{4-6} ;(3)$ blending of BMIs with epoxies ${ }^{7-9}$; (4) copolymerization of BMIs with olefinic compounds via the Diels-Alder reaction $^{10-12}$; and (5) use of aromatic dicyanate esters to blend with BMIs ${ }^{13,14}$. However, the first three methods have the disadvantage of lowering the glass transition temperature $\left(T_{\mathrm{g}}\right)$ of the cured resins. Although the fourth and fifth methods have less adverse effects on the high temperature properties and processability, BMIs have a tendency to recrystallize from the mixture with aging ${ }^{15}$ and the prepared resin systems often show inconsistent properties in different batch.

Recently, we suggested a novel method to modify the BMI resins by reacting with various amounts of allylamine $^{16,17}$. The prepared BMI/allylamine (BMI/A) adducts have a much lower degree of crystallinity and possess good mechanical properties. Their $T_{\mathrm{g}}$ can be as high as $340^{\circ} \mathrm{C}$ if the proper type of BMI and amount of allylamine are used. However, by carefully examining the

\footnotetext{
* To whom correspondence should be addressed
}

reaction paths between the BMI and allylamine, we found that they are strongly dependent on the type of BMI resin. For example, 4,4'-bismaleimidodiphenylmethane (BDM) reacts with allylamine through the Michael addition reaction, whereas 3,3'-bismaleimidodiphenylsulfone (3-BDS) reacts with allylamine by amidation through the cleavage of an imide-ring $\mathrm{C}-\mathrm{N}$ bond ${ }^{18}$.

To further investigate the reaction paths between BMI and allylamine, we have chosen 4,4'-bismaleimidodiphenylether (BDE) and 4,4'-bismaleimidodiphenylsulfone (4-BDS) in addition to BDM and 3-BDS, to react with various amounts of allylamine. The cure, mechanical and high temperature properties of the prepared BMI/A adducts will be compared.

\section{EXPERIMENTAL}

\section{Preparation of BMI monomers}

BDM, BDE, 3-BDS and 4-BDS were prepared from maleic anhydride (Janssen Chimica) and 4,4'-diaminodiphenylmethane (Janssen Chimica), 4,4'-diaminodiphenylether (Tokyo Kasei), 3,3'-diaminodiphenylsulfone (Tokyo Kasei), and 4,4'-diaminodiphenylsulfone (Tokyo Kasei), respectively, by the methods described in ref. 16 with some modifications. In general, a solution of diamine $(1 \mathrm{~mol})$ in acetone was added dropwise into a flask which had been already charged with a solution of maleic anhydride $(2 \mathrm{~mol})$ in acetone. The addition was performed at ice-temperature under a nitrogen atmosphere, and then the flask was stirred overnight at room temperature. The resulting insoluble amic acid was filtered, 
washed with acetone, and dried under vacuum. Cyclization of the amic acid $(0.5 \mathrm{~mol})$ to prepare the BDM and $\mathrm{BDE}$ was carried out in an acetone solution containing acetic anhydride $(1 \mathrm{~mol})$, triethylamine $(0.3 \mathrm{~mol})$, and nickel(II) acetate tetrahydrate $(0.03 \mathrm{~mol})$ at room temperature for $\approx 50 \mathrm{~h}$ until the product precipitated. Cyclization to prepare the 3-BDS and 4-BDS was carried out in an acetone solution containing amic acid $(0.5 \mathrm{~mol})$, acetic anhydride $(1 \mathrm{~mol})$, and sodium acetate $(0.3 \mathrm{~mol})$ at $50^{\circ} \mathrm{C}$ overnight until they precipitated. All the precipitates were collected and washed with a sodium bicarbonate solution until free from acetic acid. Finally, the $\mathrm{BDM}$ and BDE products were recrystallized from $\mathrm{CHCl}_{3} / \mathrm{MeOH}(1: 1$ by volume) solution, whereas the 3-BDS and 4-BDS were recrystallized from methanol.

\section{Preparation of N-phenylmaleimide (PMI) model compound}

PMI model compound was prepared from maleic anhydride and aniline (Hayashi Pure Chemical) based on a method outlined in US Patent $2,444,536^{19}$. A solution of aniline ( $1 \mathrm{~mol}$ ) in ethyl ether was added dropwise to the flask which had been already charged with a solution of maleic anhydride $(1 \mathrm{~mol})$ in ethyl ether. The addition was performed at ice-bath temperature under a nitrogen atmosphere. Afterwards, the flask was stirred for $1 \mathrm{~h}$ at room temperature and then cooled to $15-20^{\circ} \mathrm{C}$. The resulting insoluble maleanilic acid was filtered, washed with ethyl ether and dried under vacuum. Cyclization of the maleanilic acid $(1 \mathrm{~mol})$ was carried out in acetic anhydride $(350 \mathrm{ml})$ containing $33 \mathrm{~g}$ of sodium acetate at $100^{\circ} \mathrm{C}$. After the maleanilic acid had completely dissolved, the reaction mixture was cooled to room temperature and then poured into $700 \mathrm{ml}$ of ice-water. The precipitated product was collected and washed three times with $300 \mathrm{ml}$ ice-water and once with $300 \mathrm{ml}$ petroleum ether. After being dried, the PMI product was recrystallized from cyclohexane.

\section{Preparation of $B M I / A$ adducts}

All of the BDM/A, BDE/A, 3-BDS/A, and 4-BDS/A adducts were prepared by the same procedure. In general, various amounts of allylamine (Tokyo Kasei) in chloroform were added dropwise into a flask which had been charged with a slurry of BMI $(0.2 \mathrm{~mol})$ in chloroform. The reaction was carried out at $40^{\circ} \mathrm{C}$ for $\approx 5 \mathrm{~h}$ under a nitrogen atmosphere until the BMI was completely dissolved and a clear solution obtained. The solution was washed with distilled water and solid products obtained by removing the chloroform under vacuum at $90^{\circ} \mathrm{C}$. To name the $\mathrm{BMI} / \mathrm{A}$ adduct prepared with different molar percentages of allylamine, we designated the product of BMI reacting with $10 \mathrm{~mol} \%$ allylamine as a $\mathrm{BMI} / 10 \%$ adduct, and so on. The molar percentage is calculated by dividing the moles of allylamine by the moles of BMI.

\section{Preparation of PMI/allylamine (PMI/A) adduct}

A solution of allylamine $(1 \mathrm{~mol})$ in chloroform was added dropwise into a flask which had been already charged with PMI $(1 \mathrm{~mol})$ in chloroform solution. The reaction was performed at $40^{\circ} \mathrm{C}$ under a nitrogen atmosphere for $2 \mathrm{~h}$. The precipitated product was then collected and washed with chloroform before being dried under vacuum.

\section{Curing of $B M I / A$ adducts}

Curing of the various $\mathrm{BMI} / \mathrm{A}$ adducts was conducted in a hot press under $1.38 \times 10^{7} \mathrm{~Pa}$ pressure for $2 \mathrm{~h}$ at $180^{\circ} \mathrm{C}$ plus $5 \mathrm{~h}$ at $220^{\circ} \mathrm{C}$.

\section{Sample characterization}

I.r. spectra of the samples were recorded on a Hitachi 270-30 Model IR with $\mathrm{KBr}$ pellets. ${ }^{1} \mathrm{H}$ and ${ }^{13} \mathrm{C}$ n.m.r. spectra were recorded on a Bruker AM-300WB Model NMR spectrometer with dimethyl sulfoxide- $d_{6}$ (DMSO- $d_{6}$ ) or $\mathrm{CDCl}_{3}$ as solvent. Mass spectra were recorded on a Finnigan Mat TSQ-46C Model mass spectrometer. Differential scanning calorimetry (d.s.c.) was performed in a Du Pont 9900-910 Model DSC. Dynamic mechanical analysis (d.m.a.) was performed in a Du Pont model 9900-983 DMA. Thermogravimetric analysis (t.g.a.) was conducted in a Du Pont Model 9900-954 TGA. All the thermal analyses were run under a nitrogen atmosphere at a heating rate of $10^{\circ} \mathrm{C} \mathrm{min}^{-1}$. The flexural properties of cured samples were measured by the three-point bending test according to ASTM-D790. Tests were run in an Instron TM tensile tester at a crosshead speed of $0.5 \mathrm{~cm} \mathrm{~min}^{-1}$.
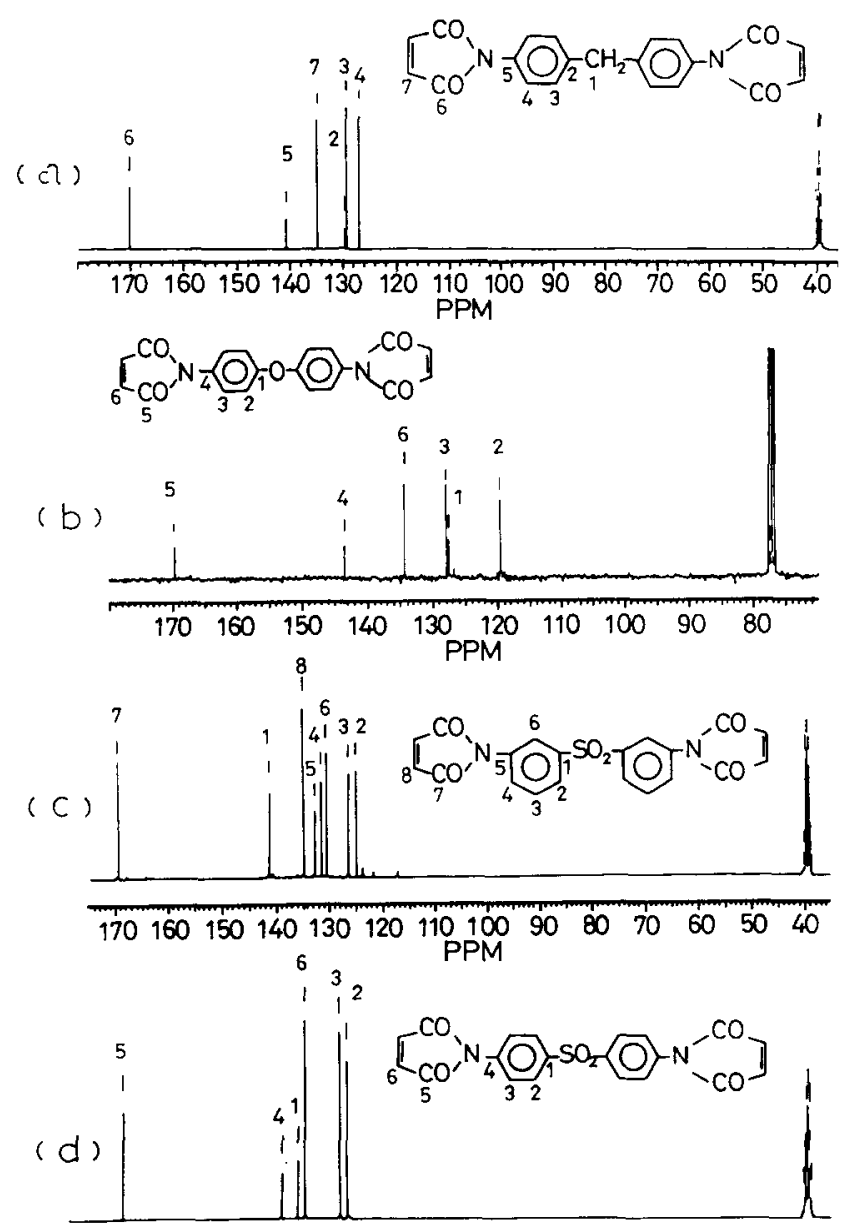

$170 \quad 160 \quad 150 \quad 140 \quad 130 \quad 120 \quad 110 \quad 100 \quad 90 \quad 80 \quad 70 \quad 60 \quad 50 \quad 40$ PPM

Figure $1{ }^{13} \mathrm{C}$ n.m.r. spectra of the prepared (a) BDM, (b) BDE, (c) 3-BDS and (d) 4-BDS 


\section{RESULTS AND DISCUSSION}

\section{Characterization of BMIs and BMI/A adducts}

${ }^{13}$ C n.m.r. spectra of the prepared BDM, BDE, 3-BDS, and 4-BDS are shown in Figure 1 , along with the assignments of each carbon. Their d.s.c. spectra shown in Figure 2 indicated that they are crystalline solids with melting points at $158,177,207$ and $248^{\circ} \mathrm{C}$, respectively. The curing peaks of BDM and BDE are broad, both appearing at $220^{\circ} \mathrm{C}$. They have been associated with the thermal homopolymerization of the resins ${ }^{16,19}$. In addition, the thermal polymerization of 3-BDS and 4BDS resins took place at 211 and $253^{\circ} \mathrm{C}$, immediately after they had become molten.

Various BMI resins were reacted with 10,50 and $200 \mathrm{~mol} \%$ allylamine to yield a series of BMI/A adducts and changes in their i.r. spectra with the amount of allylamine are shown in Figure 3. Interestingly, by comparison between four different types of BMI/A adducts, we found the $\mathrm{BDM} / \mathrm{A}$ adducts were formed exclusively by the Michael addition reaction; the peaks at $3140 \mathrm{~cm}^{-1}$ contributed by the double bonds of the maleimido groups disappeared when BDM was reacted with $200 \mathrm{~mol} \%$ allylamine. Also, the intensity of the peaks at 920 and $3300 \mathrm{~cm}^{-1}$ contributed by the allyl groups and secondary amino groups gradually increased with the amount of allylamine. On the other hand, 3-BDS/A and 4-BDS/A adducts did not undergo Michael addition reactions as indicated by insignificant change of the peaks at $3140 \mathrm{~cm}^{-1}$. The imido peaks at 1720 and $1780 \mathrm{~cm}^{-1}$ completely disappeared and were replaced by the amido peaks at $1640 \mathrm{~cm}^{-1}$ when BDS reacted with $200 \mathrm{~mol} \%$ allylamine.

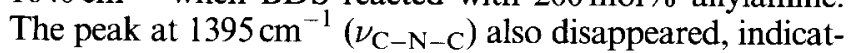
ing that the imido ring had opened. When $\mathrm{BDE}$ reacted with $200 \mathrm{~mol} \%$ allylamine, the imido rings were not completely opened as indicated by the i.r. spectra (Figure 3). It is believed that small amount of allylamine still participated in the Michael addition reactions.

Michael addition reactions between BMIs and aromatic amine have been well studied and documented ${ }^{4-6}$. However, how the allylamine opened the imido ring was not understood until we used PMI as a model compound to react with allylamine in another study ${ }^{18}$. It has been shown that the amino group of allylamine attacked one of the carbonyl groups of maleimido ring, and yielded two amido groups along with the cleavage of imide-ring

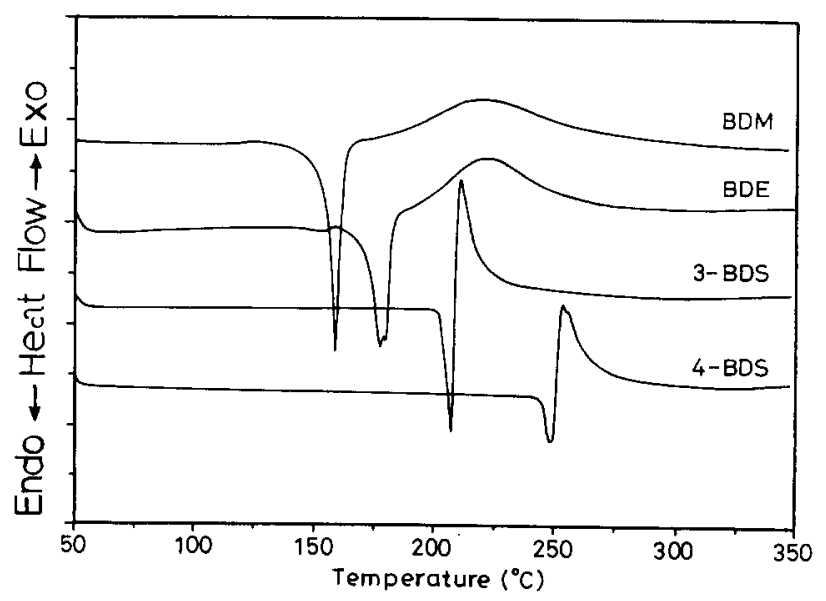

Figure 2 D.s.c. spectra of the prepared BDM, BDE, 3-BDS and 4-BDS
$\mathrm{C}-\mathrm{N}$ bond. The chemical structure of the PMI/A adduct was suggested to be as follows:

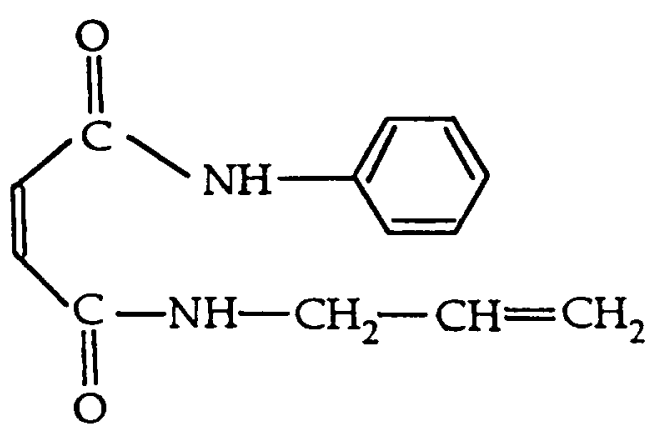

The presence of two amido groups in the PMI/A adduct has been verified by the ${ }^{1} \mathrm{H}$ n.m.r. spectrum, for the NH's are exchangeable with $\mathrm{D}_{2} \mathrm{O}^{18}$. In this study we confirmed the chemical structure by $2 \mathrm{D}$ n.m.r. shown in Figures 4 and 5 . The $2 \mathrm{D}$ representation is the line-to-line correspondence between ${ }^{1} \mathrm{H}$ and ${ }^{13} \mathrm{C}$ splitting. Using this method, the chemical structure of the PMI/A adduct can be easily determined from the $2 \mathrm{D}{ }^{1} \mathrm{H}-{ }^{13} \mathrm{C}$ correlated spectroscopy (COSY) shown in Figure 4. For example, the $\mathrm{NH}$ peak at $8.6 \mathrm{ppm}$ of the ${ }^{1} \mathrm{H}$ n.m.r. spectrum was correlated with the peaks of two neighbouring carbons at 41.1 and $163.3 \mathrm{ppm}$ of the ${ }^{13} \mathrm{C}$ n.m.r. spectrum, whereas the NH peak at $10.4 \mathrm{ppm}$ was correlated with those at 119.3 and $162.2 \mathrm{ppm}$ of the ${ }^{13} \mathrm{C}$ n.m.r. spectrum. Thus, two amido groups in the PMI/A molecule can be determined. Further verification was obtained from the distortionless enhancement by polarization transfer (DEPT) experiment, in which the carbon peaks contributed by $\mathrm{CH}_{2}$ reversed their direction and the carbons without any proton attached did not appear in the DEPT ${ }^{13}$ C n.m.r. spectrum (see Figure 5). Thus, the carbon peaks in the ${ }^{13} \mathrm{C}$.m.r. spectrum of the PMI/A adduct were assigned from the $2 \mathrm{D}$ n.m.r. The i.r. spectrum of PMI/A has been reported in Figure $6^{18}$. The peaks at 1650 and $3290 \mathrm{~cm}^{-1}$ contributed by the amido groups are similar to those in the i.r. spectrum of $\mathrm{BDS} / 200 \% \mathrm{~A}$ adducts (see Figure 3). Therefore, it is clear that the amino group of allylamine has opened the imido ring of $\mathrm{BDS} / \mathrm{A}$ adducts by amidation during the adduction reaction.

So far, two reaction paths between the allylamine and BMI have been found; one is the Michael addition reaction and the other is the amidation through the cleavage of an imide-ring $\mathrm{C}-\mathrm{N}$ bond. Michael addition reaction found for BDM reacting with allylamine might be associated with the electron donor characteristic of a methylene group inbetween two phenyl rings, which deactivates the imido-ring opening reaction and favours the Michael addition reaction. On the other hand, the sulfone group of BDS is an electron acceptor which activates the imido-ring opening reaction.

\section{Curing of $B M I / A$ adducts}

Figure 7 shows the d.s.c. spectra of various BMIs adducted with 10,50 and $200 \mathrm{~mol} \%$ allylamine. It can be seen that the addition of $10 \mathrm{~mol} \%$ allylamine significantly reduced or completely erased the crystallinity of the BMIs. The initial cure temperature also shifted to 

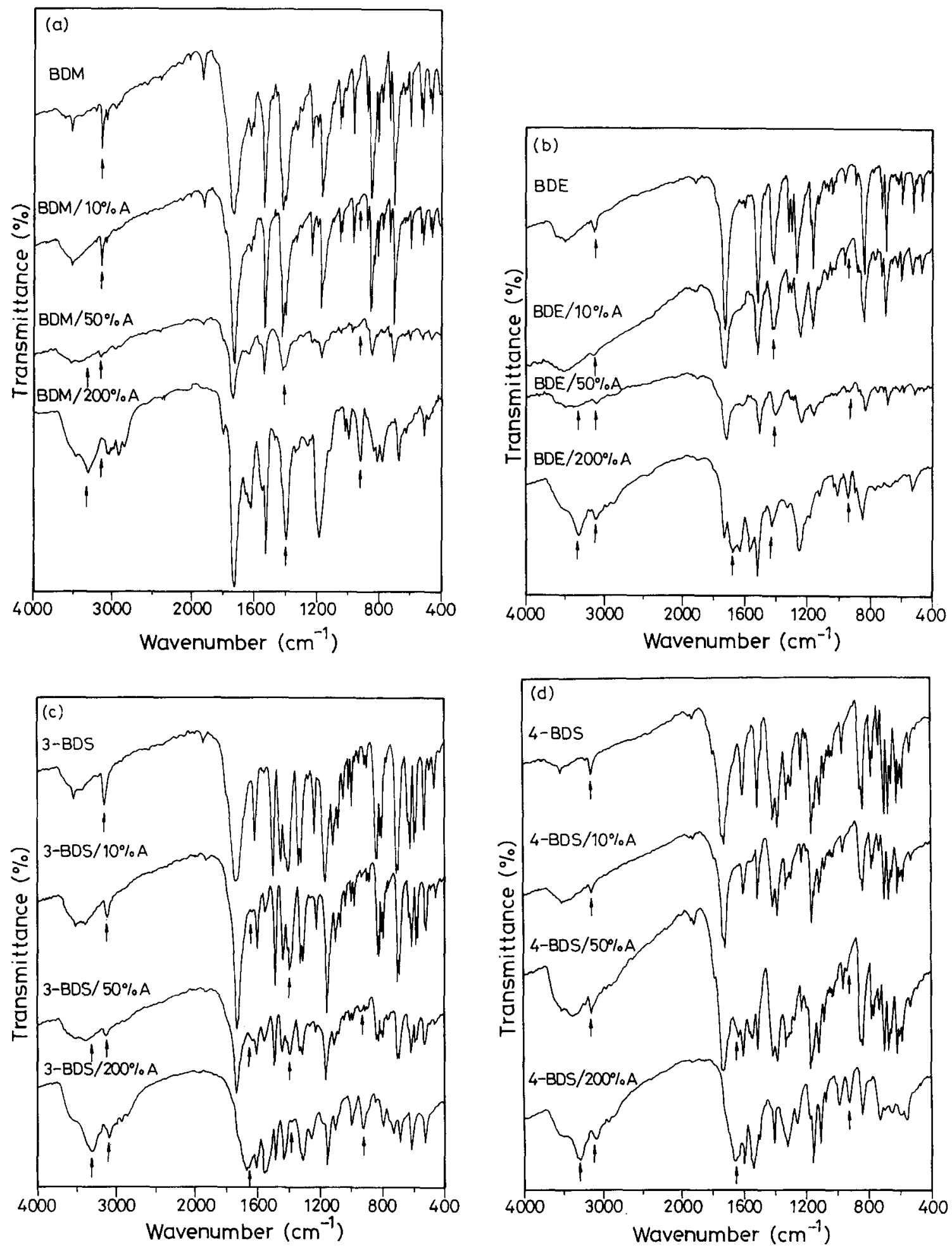

Figure 3 I.r. spectra of (a) BDM, (b) BDE, (c) 3-BDS and (d) 4-BDS, and their adducts with 10, 50 and 200\% allylamines

lower temperature (compared to Figure 2). Except for the 4-BDS/A adducts, the accelerated cure reactions of $\mathrm{BDM} / 10 \% \mathrm{~A}, \mathrm{BDE} / 10 \% \mathrm{~A}$ and $3-\mathrm{BDS} / 10 \% \mathrm{~A}$ were due to the accelerated homopolymerization of $\mathrm{BDM}, \mathrm{BDE}$, and 3 -BDS portions under the influence of the adducted allylamines. Homopolymerization was consistent with the mass spectra recorded. For instance, a mass spectrum of cured $\mathrm{BDM} / 10 \% \mathrm{~A}$ adduct has a base peak at $m / z 358$, equivalent to the molecular weight of BDM (see Figure $8)$. In this experiment, the specimens were inserted directly into the mass spectrometer and the pyrolysis was performed close to the ion source. Thus, the appearance of the peak at $m / z 358$ is consistent with depolymerization in the pyrolytic stage and indicative of the incorporation of this unit in the polymer. Similar peaks were also found in the mass spectra of the homopolymerized $\mathrm{BMI}^{18}$. The accelerated effects of adducted allylamine on the polymerization of BDM were believed to be attributable to the "charge transfer complex' effect ${ }^{20}$ in view of the fact that the allyl groups from the adducted allylamine is an electron-rich olefin and the double bonds of the maleimido groups are an electron poor olefin. 


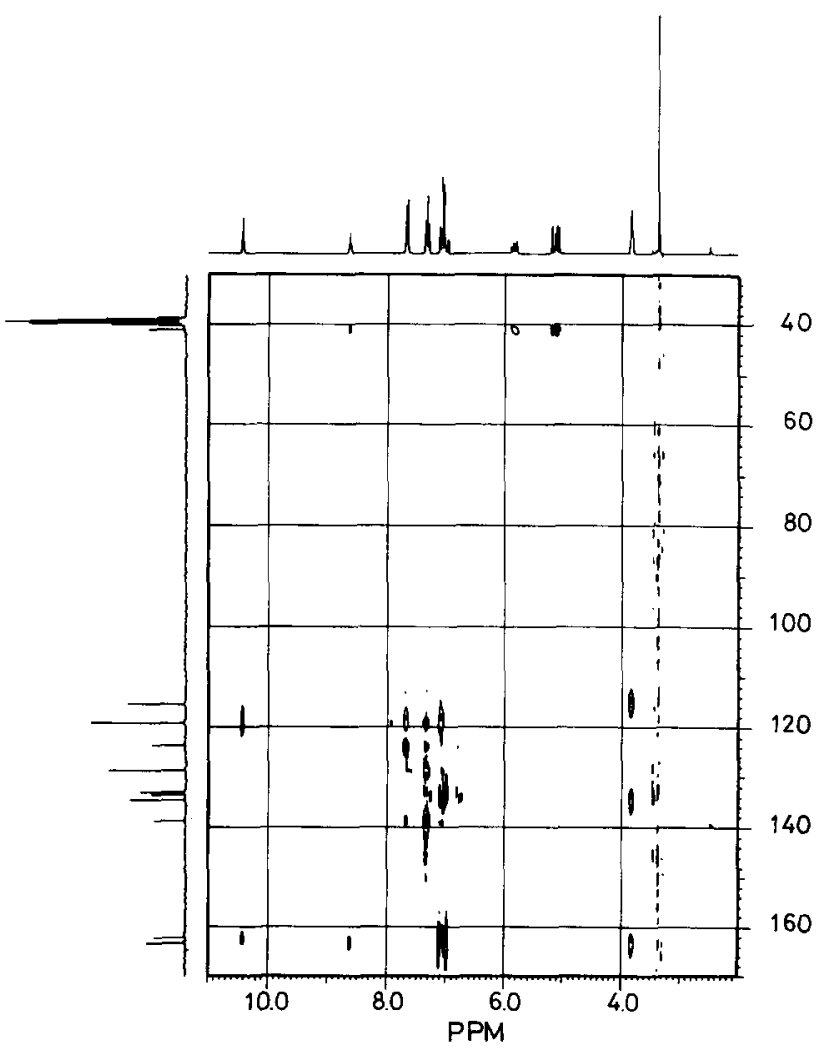

Figure $42 \mathrm{D}{ }^{1} \mathrm{H}-{ }^{13} \mathrm{C}$ correlated spectrum (COSY) of the prepared PMI/A adduct
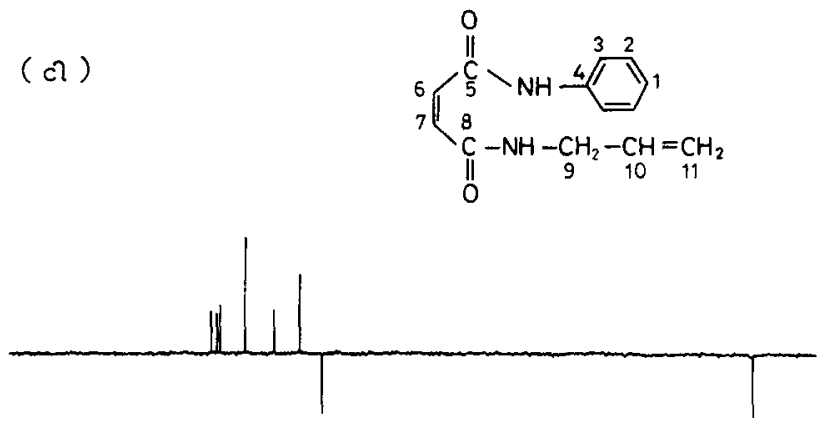

(b)

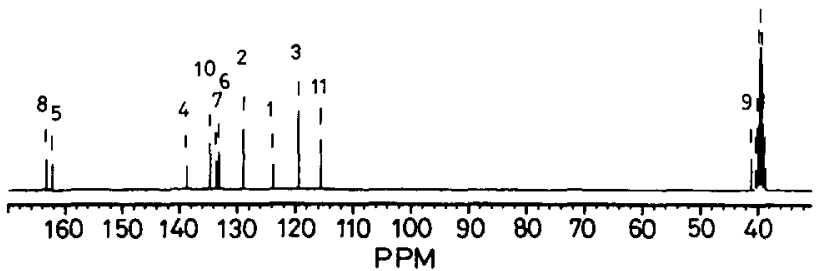

Figure 5 (a) DEPT and (b) ${ }^{13}$ C n.m.r. spectra of the prepared PMI/A adduct

However, this would not be the case for $3-\mathrm{BDS} / 10 \% \mathrm{~A}$ adducts, because the adducted allylamines were prone to cleavage from the $3-\mathrm{BDS} / \mathrm{A}$ adducts at $170-180^{\circ} \mathrm{C}$ as indicated by their t.g.a. and i.r. spectra.

A small drop in the weight of $3-\mathrm{BDS} / 10 \% \mathrm{~A}$ adduct was observed as shown in Figure 9a, indicating that some degradation took place at a temperature slightly lower than the cure temperature. I.r. spectra of the 3-BDS/ $200 \% \mathrm{~A}$ adduct after heating at $180^{\circ} \mathrm{C}$ for $30 \mathrm{~min}$

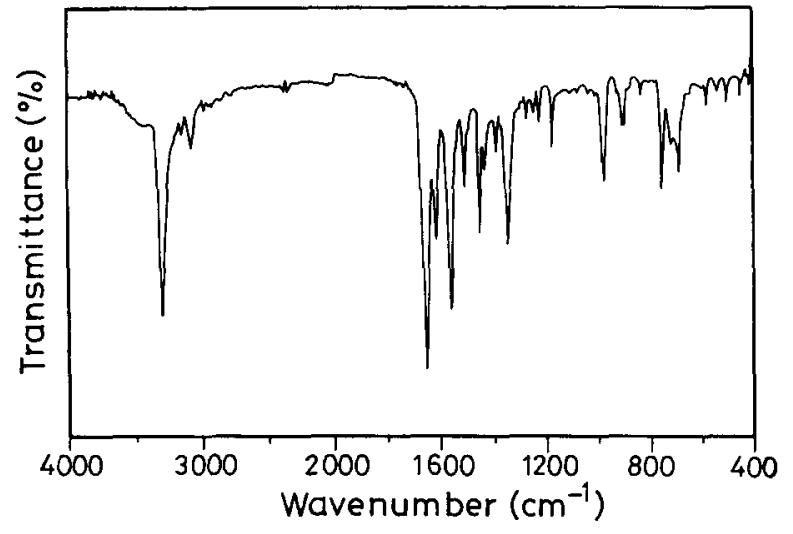

Figure 6 I.r. spectrum of the prepared PMI/A adduct ${ }^{18}$

revealed that the degradation was due to the cleavage of allylamine groups along with the recovery of the maleimido groups (see Figure 10). The peaks at 1720 and $1420 \mathrm{~cm}^{-1}$ contributed by the imido ring reappeared, whilst the peaks at $1660 \mathrm{~cm}^{-1}$ contributed by the amido groups and at $920 \mathrm{~cm}^{-1}$ contributed by the allyl groups were reduced in their intensity. Since the cleaved allylamino groups have chances to possess free radicals and/ or ionic charges, they might initiate the homopolymerization of $3-\mathrm{BDS}$ in the $3-\mathrm{BDS} / 10 \% \mathrm{~A}$ adduct at lower temperature. Due to the similarity of the chemical structure between the 3-BDS and 4-BDS, the allylamino groups of $4-\mathrm{BDS} / 10 \% \mathrm{~A}$ adducts were also cleaved prior to the cure temperature. The cure peak of 4 -BDS $/ 10 \% \mathrm{~A}$ adduct was barely found at $\approx 190^{\circ} \mathrm{C}$ but grew larger as the amount of adducted allylamine was increased. The cleaved allylamines are not able to initiate the homopolymerization of 4-BDS, because the double bonds of 4-BDS are quite unreactive as indicated by their polymerization at much higher temperature $\left(\approx 250^{\circ} \mathrm{C}\right)$. Thus, the cleaved allylamines might react with each other through double bonds of the allyl groups and contributed to the cure peaks at $170-190^{\circ} \mathrm{C}$.

When the amount of allylamine to adduct BDM was increased to $50 \mathrm{~mol} \%$, the melting peak further shifted to lower temperature and the curing peak became broader. However, the heat of exotherm is $200 \mathrm{Jg}^{-1}$ similar to that of $\mathrm{BDM} / 10 \% \mathrm{~A}$, indicating that the allyl groups also participated in the curing reactions through the "charge transfer complex' mechanism. When the amount of allylamine was increased to $200 \mathrm{~mol} \%$, the initial curing peak was significantly reduced. However, the other peak, which appeared at $\approx 245^{\circ} \mathrm{C}$, was believed to be associated with the reactions of cleaved allylamines, because the adducted allylamines were cleaved at a similar temperature to that seen in their t.g.a. thermogram shown in Figure $9 b$. On the other hand, for the 3-BDS/A adducts, the allylamino groups were cleaved along with the recovery of imido ring of $3-\mathrm{BDS}$ at $170^{\circ} \mathrm{C}$, prior to the cure reactions. When it is in small quantity $(\leq 50 \mathrm{~mol} \%)$, the cleaved allylamine will accelerate the homopolymerization of 3-BDS through the maleimide double bonds. When the cleaved allylamines are plentiful, they have more chance to encounter each other and polymerize by themselves. As a result, the initial cure peak was still strong and appeared at a lower temperature when the allylamine was increased to $200 \mathrm{~mol} \%$. Since the cleaved 

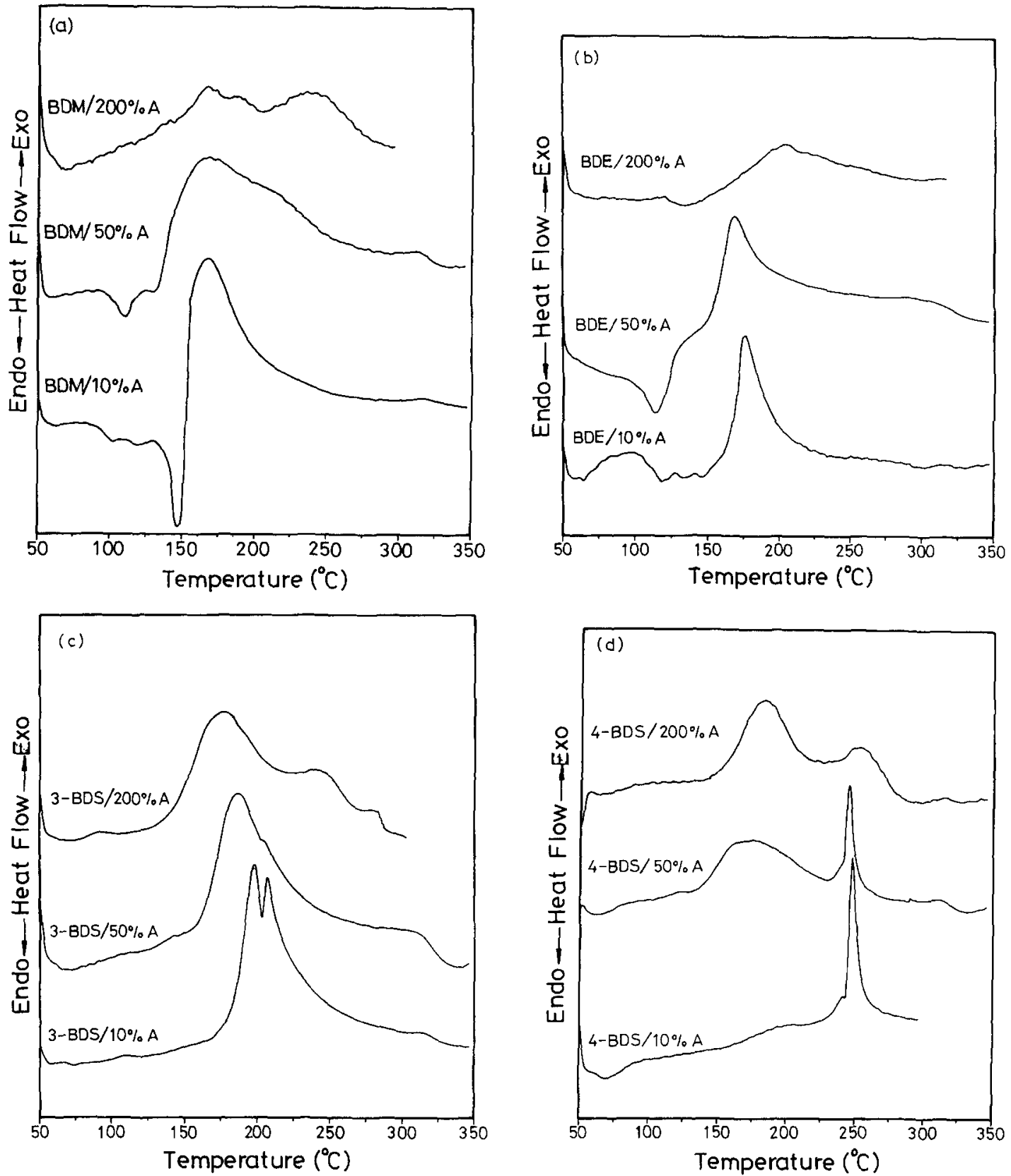

Figure 7 D.s.c. spectra of (a) BDM/A, (b) BDE/A, (c) 3-BDS/A and (d) 4-BDS/A adducts with 10,50 and 200 mol $\%$ allylamines

allylamines had a better chance to react with each other, they did not vaporize off at the cure temperature range as indicated in the t.g.a. thermogram of the $3-\mathrm{BDS} / 200 \% \mathrm{~A}$ adduct (Figure 9a).

In the case of $\mathrm{BDE} / \mathrm{A}$ adducts, their cure peak became extremely broad when BDE was adducted with $200 \mathrm{~mol} \%$ allylamine (Figure $7 \mathrm{~b}$ ). Similar to the BDM/ $200 \% \mathrm{~A}$ adduct, the previous cure peak at $170-180^{\circ} \mathrm{C}$ was reduced and the major cure peak, which appeared at $200^{\circ} \mathrm{C}$, was believed to be associated with the reactions of cleaved allylamine. Since the allylamino groups of $\mathrm{BDE} / \mathrm{A}$ adducts were partly from a Michael addition reaction and partly from amidation, their cleavage temperature is presumably higher than that of the BDS/A adducts but lower than $\mathrm{BDM} / \mathrm{A}$ adducts.

Properties of the cured BMI/A adducts

Although the BMI resins are able to undergo thermal

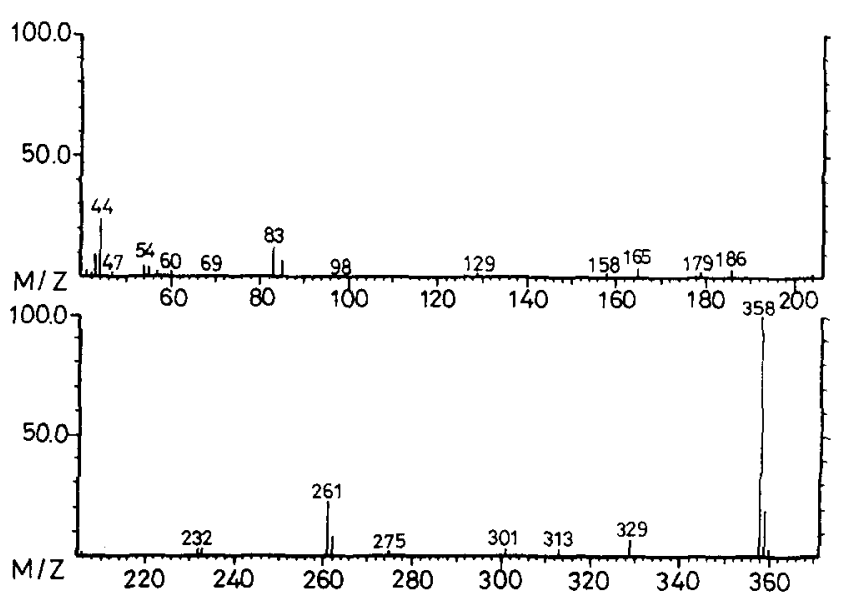

Figure 8 A typical mass spectrum of cured $\mathrm{BDM} / 10 \% \mathrm{~A}^{16}$ 

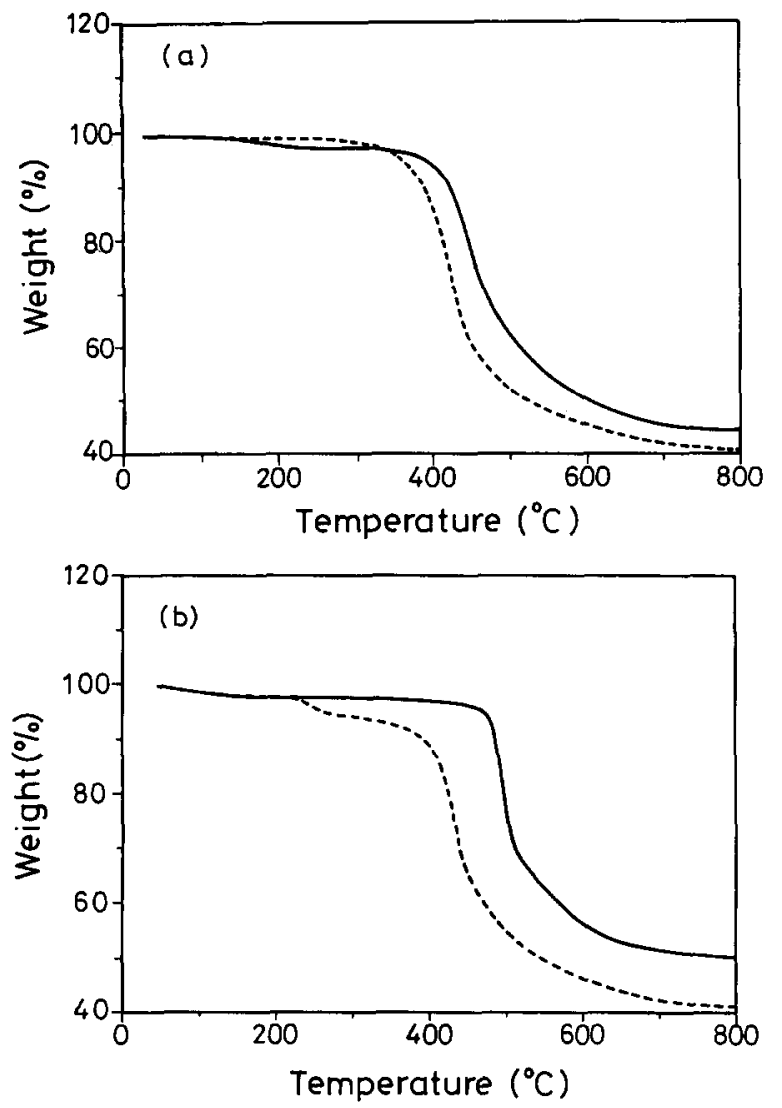

Figure 9 T.g.a. thermograms of the allylamine-adducted (a) 3-BDS and (b) BDM, both adducted with $\left(-{ }_{-}\right) 10 \mathrm{~mol} \%$ and $(--)^{-} 200 \mathrm{~mol} \%$ allylamines

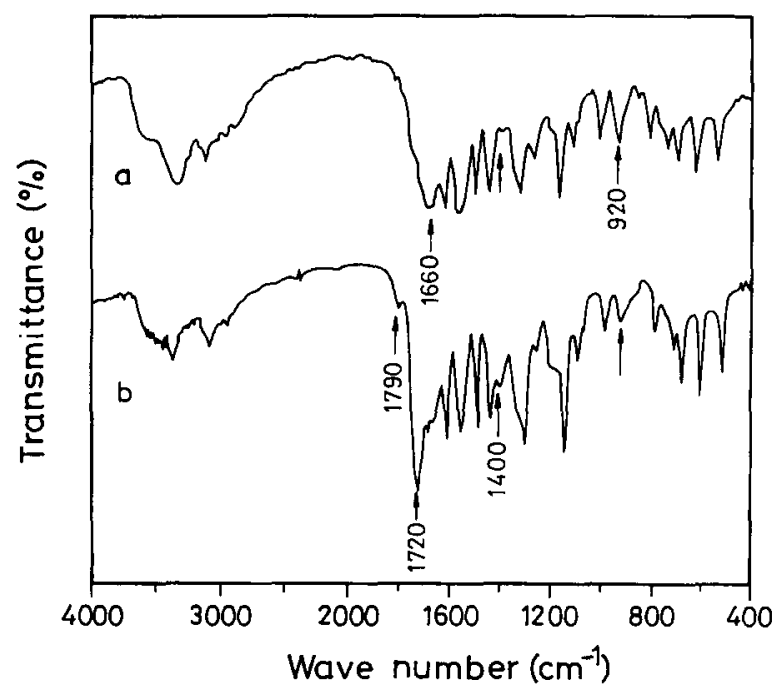

Figure 10 I.r. spectra of the 3-BDS $/ 200 \% \mathrm{~A}$ adducts: (a) without cure and (b) cured at $180^{\circ} \mathrm{C}$ for $30 \mathrm{~min}$

homopolymerization, they are too brittle to be moulded as a specimen for mechanical tests. Moreover, 3-BDS/ $200 \% \mathrm{~A}$ and $4-\mathrm{BDS} / 200 \% \mathrm{~A}$ adducts could not be moulded either, because part of the cleaved allylamines which did not participate in the curing reactions would create voids. Excluding the above, the $T_{\mathrm{g}}$ of cured (or moulded) BMI/A adducts under study, defined by the loss modulus peak of d.m.a. spectra, is measured and shown in Figure 11. Apart from the cured 3-BDS/A adducts, the BMI adducted with $10 \mathrm{~mol} \%$ allylamine

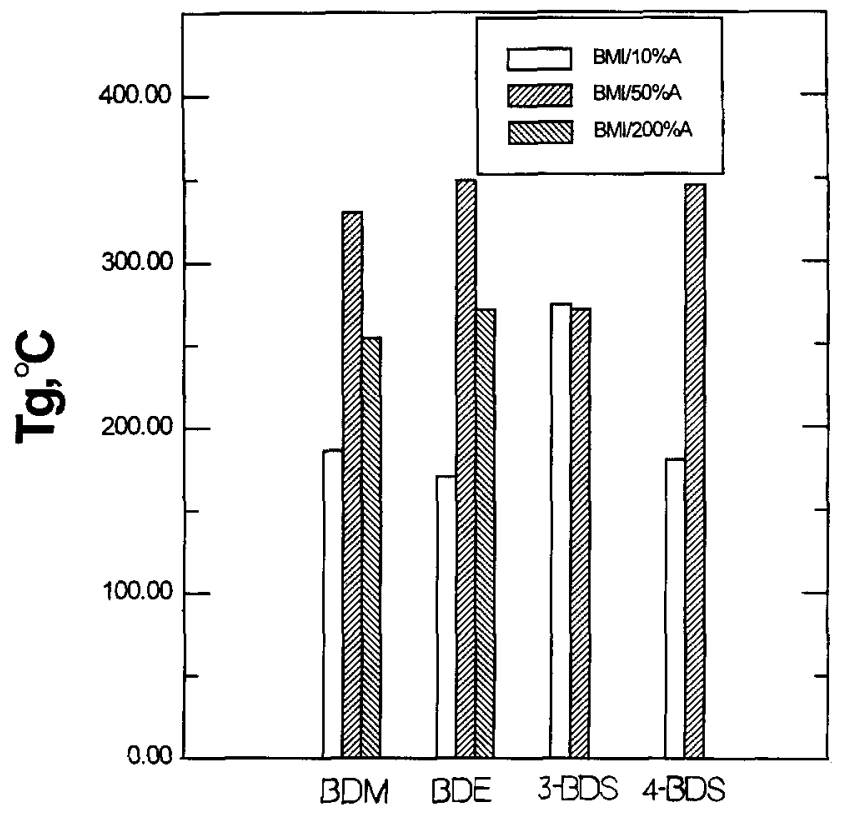

Figure $11 T_{\mathrm{g}}$ of the cured BDM/A, BDE/A, 3-BDS/A, and 4-BDS/A adducts with 10,50 and $200 \mathrm{~mol} \%$ allylamines

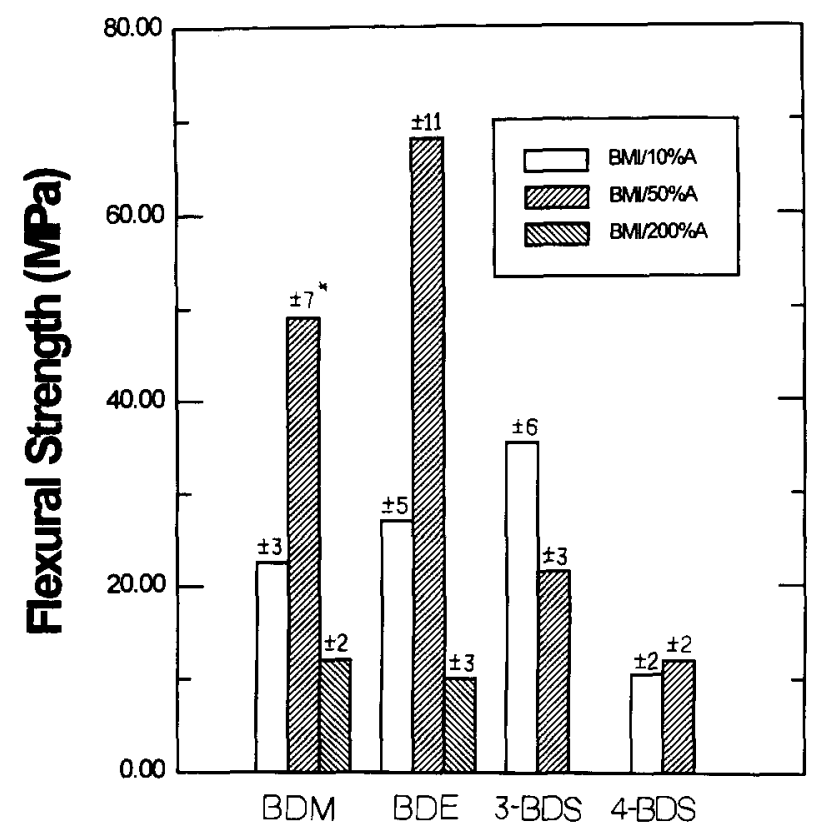

Figure 12 Flexural strength of the cured BDM/A, BDE/A, 3-BDS/A and 4-BDS/A adducts with 10,50 and $200 \mathrm{~mol} \%$ allylamines

have the lowest $T_{\mathrm{g}}$. However, when the amount of adducted allylamine was increased to $50 \%$, the $T_{\mathrm{g}}$ reached its highest value, more than $330^{\circ} \mathrm{C}$ (except for cured 3-BDS/A adduct). When it was further increased to $200 \%$, the $T_{\mathrm{g}}$ of $\mathrm{BDM} / 200 \% \mathrm{~A}$ and $\mathrm{BDE} / 200 \% \mathrm{~A}$ adducts was reduced because of the lesser amount of curing (Figure 7). A similar trend was also found for the flexural strength of cured BMI/A adducts (except for cured 3-BDS/A adduct), where that of the cured BMI/ $50 \% \mathrm{~A}$ adducts was the highest (as shown in Figure 12). Although the similar heat exotherm during cure was found between $\mathrm{BDM} / 10 \% \mathrm{~A}$ (and $\mathrm{BDE} / 10 \% \mathrm{~A}$ ) and $\mathrm{BDM} / 50 \% \mathrm{~A}$ (and $\mathrm{BDE} / 50 \% \mathrm{~A}$ ), their $T_{\mathrm{g}}$ and flexural strength differ tremendously. It seems that the adducted 


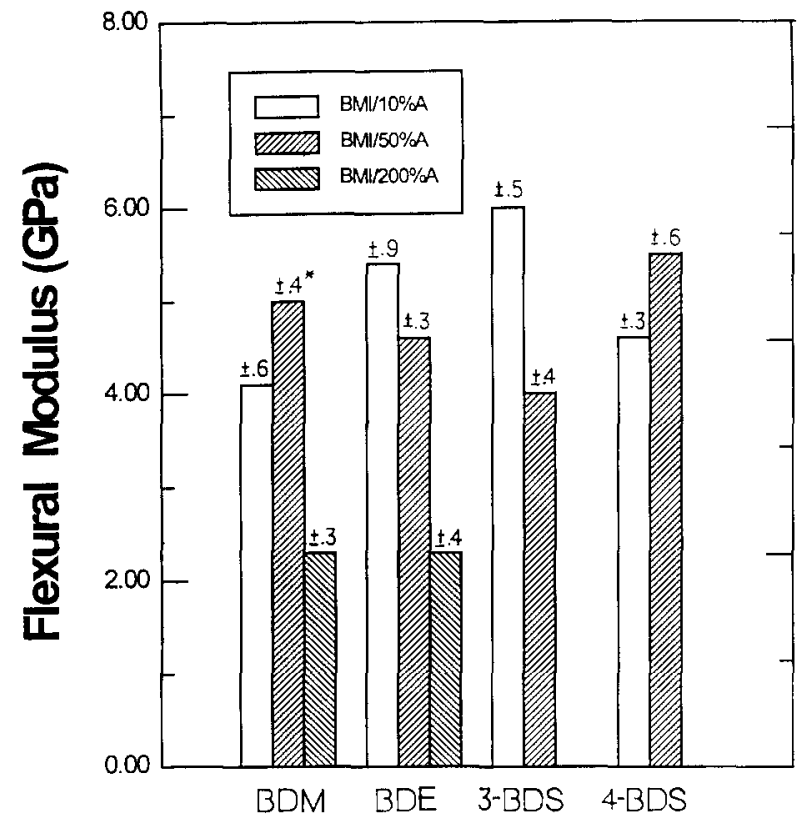

Figure 13 Flexural moduli of the cured BDM/A, BDE/A, 3-BDS/A and $4-B D S / A$ adducts with 10,50 and $200 \mathrm{~mol} \%$ allylamines

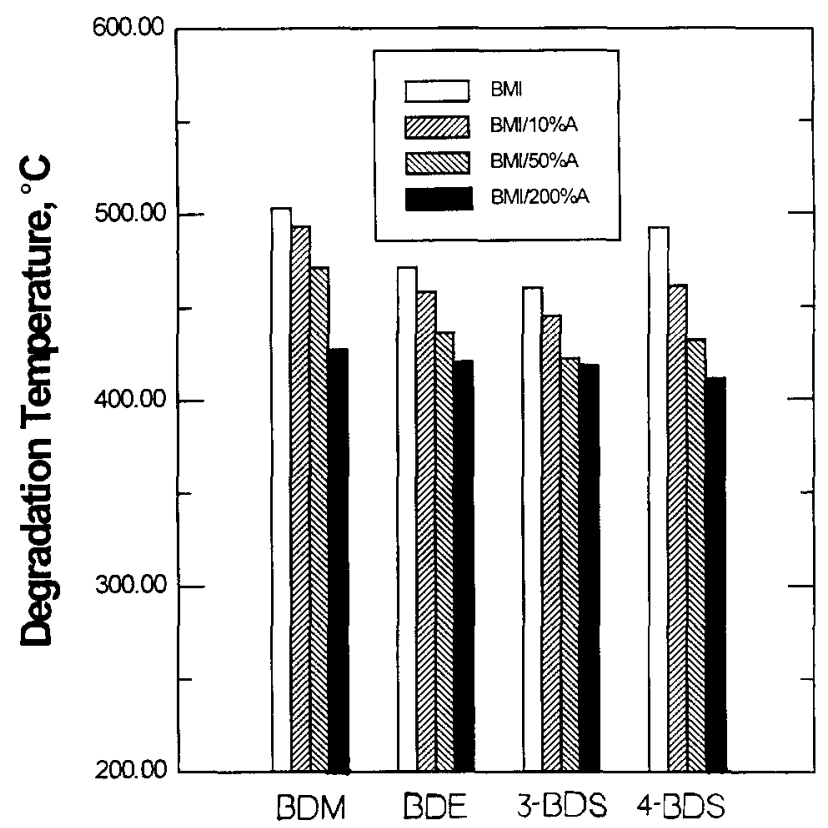

Figure 14 Degradation temperatures of BDM, BDE, 3-BDS and 4-BDS, and their adducts with 10,50 and $200 \mathrm{~mol} \%$ allylamines

allylamines in the $\mathrm{BMI} / 50 \% \mathrm{~A}$ adducts were able to act as a cross-linker to increase the crosslinking density. On the other hand, all the cured BMI/A adducts are of a brittle material, as indicated by their high flexural moduli shown in Figure 13. The lowest flexural moduli found for the cured $\mathrm{BDM} / 200 \% \mathrm{~A}$ and $\mathrm{BDE} / 200 \% \mathrm{~A}$ might be a result of the low extent of cure and the possession of large quantity of unreacted allylamino groups.

The degradation temperature $\left(T_{\mathrm{d}}\right)$ of a series of BMI resins and their BMI/A adducts measured by t.g.a. at the maximum degradation rate is shown in Figure 14. The BDM resin has the highest $T_{\mathrm{d}}$ of $503^{\circ} \mathrm{C}$. When it was adducted with $50 \%$ allylamine, the $T_{\mathrm{d}}$ only dropped to $471^{\circ} \mathrm{C}$. The $T_{\mathrm{d}}$ of the $\mathrm{BDE}$ resin was $471^{\circ} \mathrm{C}$. When it was adducted with $50 \%$ allylamine, the $T_{\mathrm{d}}$ dropped to $436^{\circ} \mathrm{C}$. In spite of this, the cured $\mathrm{BDE} / 50 \% \mathrm{~A}$ adduct has the highest $T_{\mathrm{g}}$ and flexural strength among all the BMI/A adducts. Although the cured $4-\mathrm{BDS} / 50 \% \mathrm{~A}$ adduct has a rather high $T_{\mathrm{g}}$ of $346^{\circ} \mathrm{C}$ and a $T_{\mathrm{d}}$ of $432^{\circ} \mathrm{C}$, its flexural strength is extremely low (see Figure 12). Therefore, comparing the thermal and mechanical properties of all the cured $\mathrm{BMI} / \mathrm{A}$ adducts under study, $\mathrm{BDM} / 50 \% \mathrm{~A}$ and $\mathrm{BDE} / 50 \% \mathrm{~A}$ adducts are superior and have the potential to be used as high temperature resin in aerospace, electronic, and other high-technology applications.

\section{CONCLUSIONS}

BDM, 3-BDS, and 4-BDS adducted with various amount of allylamines were synthesized by one of the following two reaction paths: Michael addition reaction for $\mathrm{BDM}$ reacting with allylamine, or amidation along with the cleavage of an imide-ring $\mathrm{C}-\mathrm{N}$ bond, found for 3-BDS and 4-BDS reacting with allylamine. Only synthesis of the BDE/A adducts underwent both reaction paths. The allylamino groups adducted by the Michael addition reaction are more stable (cleavage at $245^{\circ} \mathrm{C}$ ), whereas those by amidation, cleaved at $170^{\circ} \mathrm{C}$ prior to the cure reactions. Three types of reaction paths for curing of various BMIs and their BMI/A adducts probably occurred: (1) thermal homopolymerization of various BMI resins through the maleimide double bonds; (2) accelerated homopolymerization of the BMI portions by the adducted or then-cleaved allylamino groups; and (3) polymerization of the already cleaved allylamines from BMI/A adducts (especially for BDS/A adducts) through the double bonds of allyl groups or with the recovered maleimido groups.

As to the properties of BMIs and their BMI/A adducts, $\mathrm{BMI}$ resins, $3-\mathrm{BDS} / 200 \% \mathrm{~A}$ and $4-\mathrm{BDS} / 200 \% \mathrm{~A}$ are too brittle to be moulded. Except for 3-BDS/A adducts, the $\mathrm{BMI} / 10 \% \mathrm{~A}$ adducts have the lowest $T_{\mathrm{g}}$ and flexural strength, whereas those of the $\mathrm{BMI} / 50 \% \mathrm{~A}$ adducts are the highest. However, with a further increase the amount of adducted allylamine to $200 \mathrm{~mol} \%$, both the $T_{\mathrm{g}}$ and flexural strength were reduced owing to the lesser amount of curing. On the other hand, the $T_{d}$ of BMI/A adducts decreased with the amount of adducted allylamine. However, the cured $\mathrm{BDM} / 50 \% \mathrm{~A}$ and $\mathrm{BDE} /$ $50 \% \mathrm{~A}$ adducts still had $T_{\mathrm{d}}$ of 471 and $436^{\circ} \mathrm{C}$, respectively. With $T_{g}>330^{\circ} \mathrm{C}$ and high flexural strength, they are believed to have a great potential for high temperature applications.

\section{ACKNOWLEDGEMENTS}

Financial support of this work by the National Science Council in Taiwan, ROC, through grant NSC84-2216E002-014 is gratefully acknowledged.

\section{REFERENCES}

1 Pater, R. H. SAMPE J. 1994, 30(5), 29

2 Rao, R. S. J. Polym. Sci., Polym. Chem. Ed. 1989, 27, 2509

3 Takeda, S., Akiyama, H. and Kakiuchi, H. J. Appl. Polym. Sci. $1988,35,1341$

4 Crivello, J. V. J. Polym. Sci., Polym. Chem. Ed. 1973, 11, 1185 
High temperature resins based on allylamine/bismaleimides: $K$. $-F$. Lin et al.

Varma, I. K., Fohlen, G. M. and Parker, J. A. J. Polym. Sci Polym. Chem. Ed. 1982, 20, 283

6 Giulio, C. D., Gautier, M. and Jasse, B. J. Appl. Polym. Sci. $1984,29,1771$

Chaudhari, M., Galvin, T. and King, J. SAMPEJ. 1985, 21(4), 17 Stenzenberger, H. D. Br. Polym. J. 1988, 20, 383

Pater, R. H. U.S. Pat. 5, 081, 198, 1992

Voit, J. F. and Seferis, J. C. J. Appl. Polym. Sci. 1988, 35, 1351 Domeier, L. A. U.S. Pat. 4, 654, 407, 1987

Lin, K. F. and Chen, C. J. Polym. Eng. Sci. 1996, 36, 21

Gigat, E. and Poter, R. Angew. Chem. Int. Ed. 1967, 6, 206
'BT Resins', Commercial Data Brochure, 2nd Ed, Mitshbishi Gas Chemical Co., June 1980

15 Stenzenberger, H. D., Roemer, W., Herzog, M., Pierce, S Canning, $\mathbf{M}$. and Fear, K. '31st International SAMPE Symposium,' 1986, p. 920

Lin, K. F. and Lin, J. S. J. Appl. Polym. Sci. 1993, 50, 1601

Lin, K. F. and Lin, J. S. J. Appl. Polym. Sci. 1994, 51, 513

Lin, K. F., Lin, J. S. and Cheng, C. H. J. Polym. Res. (submitted) Searle, N. E. U.S. Pat. 2, 444, 536, 1948

Stevens, M. P. 'Polymer Chemistry-An Introduction', Addison Wesley, Reading, Mass., 1975, p. 128 